\title{
Gobernanza participativa,
}

la experiencia de Barcelona*

Fecha de recepción: 13 de agosto de 2019 | Fecha de aprobación: 18 de septiembre de 2019 | Fecha de publicación: 29 de mayo de 2020

María de Lourdes Flores Lucero

Benemérita Universidad Autónoma de Puebla,

México

Resumen El Gobierno de Barcelona cuenta desde hace más de tres décadas con experiencia en participación social para el mejoramiento de la ciudad, situándola como una de las ciudades vanguardistas en materia de gobernanza participativa (GP). No obstante, a pesar de que Barcelona cuenta con un marco reglamentario, metodologías, espacios para la participación y acciones gubernamentales para promover la participación social, alcanzar una participación colectiva significativa todavía se vislumbra como una meta distante. El objetivo de este trabajo es aportar a la reflexión sobre el desarrollo de condiciones que impulsan la participación social desde las instituciones, para fortalecer la GP, a través de la experiencia en Barcelona. Aquí se destaca la necesidad de mejorar los canales e instrumentos oficiales para aumentar la influencia de la participación social en la decisión política y asegurar el involucramiento de los sectores más vulnerables. La metodología es cualitativa y está basada en el enfoque teórico sobre gobernanza participativa y gobierno abierto, y utiliza las técnicas de entrevistas semidirigidas, el análisis documental y los recorridos de campo. 


\section{Participatory Governance,}

the Barcelona Experience

Abstract For more than three decades the Government of Barcelona has had the experience of social participation intended to improve the city, which has positioned Barcelona as one of the most avant-garde cities regarding the participatory governance (PG). However, despite Barcelona has a regulatory framework, methodologies, participatory spaces and governmental actions intended to foster the social participation, there is a long way to go in order to achieve the goal of a significant collective participation. This work aims to contribute to the reflection on the development of the conditions required to promote the social participation from the institutions, in order to strengthen the PG based on the Barcelona experience. The work underlines the need to improve the official channels and instruments in order to enhance the influence of social participation on the political decisions and to ensure that the most vulnerable population be involved. The methodology is qualitative and is based on the theoretical approach to the participatory governance and open government. It uses the techniques of semi-guided interviews, documentary analysis and tours out in the field.

Keywords: participatory governance, social participation, open government, Barcelona

\section{Governança participativa,}

a experiência de Barcelona

Resumo O Governo de Barcelona possui experiência em participação social para o melhoramento da cidade há mais de três décadas, posicionando-a como uma das cidades vanguardistas em matéria de governança participativa (GP). No entanto, a pesar de Barcelona ter uma estrutura regulatória, metodologias, espaços para a participação e açóes governamentais para promover a participação social, alcançar uma participação coletiva significativa ainda vislumbra-se uma meta longínqua. O objetivo deste trabalho é contribuir para a reflexão sobre o desenvolvimento de condiçóes que impulsionam a participação social desde as instituiçóes, para fortalecer a GP, através da experiência em Barcelona. Aqui, destaca-se a necessidade de melhorar os canais e instrumentos oficiais para aumentar a influencia da participação social na decisão política e garantir o envolvimento dos setores mais vulneráveis. A metodologia é qualitativa e baseada no enfoque teórico sobre governança participativa e governo aberto, e usa as técnicas de entrevista semi-dirigida, análise documental e visitas de campo.

Palavras-chave governança participativa, participação social, governo aberto, Barcelona 


\section{Introducción}

La participación social, impulsada desde los años ochenta en Barcelona, ha ido adquiriendo protagonismo en los discursos oficiales y se ha institucionalizado para fortalecer la gobernanza participativa (GP). No obstante, pasar de los modelos de gestión jerárquicos preestablecidos a unos más negociados y cooperativos no es una tarea fácil para las instituciones gubernamentales. Bajo la idea de impulsar la participación social, se han desarrollado normas e instituciones que con el paso de los años han experimentado avances, pero también tropiezos, ya sea por el unilateral enfoque técnico, por la multiplicación descontrolada de los órganos de participación, por la falta de continuidad de acciones entre las administraciones, etc.

Los esfuerzos del Gobierno barcelonés por construir una ciudad a partir de la participación social lo llevan a ser un ejemplo de progreso en el fomento a la cultura de la participación y en sentar las bases de una nueva gobernanza donde las decisiones se tomen de manera conjunta y negociada a través de la acción coordinada y organizada de los diferentes grupos de actores y mediante redes de ciudades (Callon, 1997; Mayntz, 2001; Pascual i Esteve, 2007). Este avance ha implicado crear las condiciones necesarias desde las instituciones públicas y sus normativas, así como impulsar los principios del gobierno abierto, basándose en acciones de transparencia y acceso a la información, para hacer más incluyentes las opiniones de la sociedad civil en la esfera de las políticas públicas.

En este marco, se analizan las principales acciones normativo-institucionales del Gobierno Barcelonés por crear condiciones para el fortalecimiento de la democracia y para instaurar nuevos modelos de gobernanza que permitan mejorar la ciudad. De esta manera, este trabajo pretende aportar a la reflexión sobre las condiciones que ha desarrollado el Ayuntamiento de Barcelona, en el marco de la gobernanza participativa, para impulsar la participación social.

La metodología de esta investigación es cualitativa y está basada en el enfoque de la gobernanza participativa, alimentado por autores como Callon (1997), Mayntz (2001), Kooiman (2004), Pascual i Esteve (2007), Blanco (2009), y el enfoque del gobierno abierto, desarrollado por la alianza del gobierno abierto a través de autores como Sandoval-Almazán (2015) y Criado y Ruvalcaba (2016). También se analizaron los estudios de Borja (1996 y 2013) y algunos documentos de la Diputació de Barcelona (2011, 2017a y 2017b), para conocer sus avances en materia de participación social. Se analizaron algunos instrumentos locales y páginas oficiales que impulsan la participación ciudadana, destacando la Ley 2/2000 de urbanismo, la Ley de Urbanismo 303/2006 donde se regula el derecho a la participación ciudadana (comentadas por la Diputació de Barcelona, 2011), el Reglamento de Participación Ciudadana (Ajuntament de Barcelona 2017a) y las páginas web Decidim Barcelona y Govern obert. Se realizaron entrevistas a funcionarios públicos, dentro de las que se destacan la del comisionado de participación y democracia activa de Barcelona y la de la técnica de participación del distrito de Ciutat Vella, para conocer su postura y los principales avances en materia de gobernanza participativa. El trabajo de campo se desarrolló, particularmente, en el distrito de Ciutat Vella, para observar algunas 
sesiones participativas con vecinos del barrio de la Barceloneta.

\section{Transitando hacia la gobernanza participativa}

Los gobiernos locales tradicionales, basados en sistemas de gestión burocráticos y jerárquicos, se han visto sometidos a presiones de cambio ante los procesos de urbanización, la globalización económica, y la emergencia de nuevos valores y nuevos referentes de identidad política entre la ciudadanía (Blanco, 2009). En este sentido, uno de los cambios más significativos ha sido el desarrollo de políticas públicas para institucionalizar la integración de los ciudadanos en los procesos de decisión política. Este cambio de paradigma ubica a una gran parte de los gobiernos locales en un proceso de transición en el que conviven los sistemas jerárquicos de gobernanza y en el que, según Callon (1997), las decisiones son predeterminadas de manera unívoca, con nuevos sistemas de cogobernanza que intentan crear las condiciones necesarias para impulsar prácticas de colaboración entre actores. De acuerdo con Kooiman (2004), esto permitirá dar solución a los problemas a través del reconocimiento de la diversidad de los intereses de los actores, la toma de desiciones sobre la relación entre las difererntes partes del problema (como sistema) y la localización de las fuentes de tensión. Es decir, se promueve el derecho a la ciudad, entendido como el derecho de los habitantes urbanos a construir, decidir y crear ciudad (Lefebvre, 1975).

\section{Condiciones para impulsar \\ una nueva gobernanza}

La nueva gobernanza contiene, según Aguilar (2006), dos dimensiones interdependientes: una valorativa (teleológica) basada en un proceso mediante el cual se define el futuro social deseado, representado por los valores de la convivencia humana dignos de ser objeto de la acción de la sociedad (la agenda social); y otra factual (causal y técnica), donde se define la división del trabajo, la distribución de la autoridad y la responsabilidad de los agentes sociales para el logro de los objetivos sociales deseados. Para alcanzar estas dimensiones, se deben de crear unas condiciones de carácter estructural que propicien esta nueva forma de gobernar. Aquí, se hace referencia a las instituciones, estructuras sociales generales, reglas y normas de conducta, esquemas de comunicación, posibilidades materiales y tecnológicas, y normas técnicas de operación (Kooiman, 2004), que, en su conjunto, permitan la elaboración de políticas públicas basadas en la interacción de actores diversos, experimentando con espacios deliberativos como consejos, mesas, foros ciudadanos, espacios virtuales, etc. (Blanco, 2009), para enfrentar los problemas socio-territoriales de las diferentes comunidades. Es importante señalar el destacado papel de los espacios de interacción, ya que, "cuanto mayor es el espacio que crea una interacción, mayor es la libertad de los actores para seleccionar los valores, objetivos e intereses que querrán procurarse posteriormente. Una interacción que crea espacios se caracteriza por una gran amplitud de acción y un alto grado de flexibilidad" (Kooiman, 2004). La ampliación de las cadenas de interacción entre los diferentes actores requiere de la multiplicación del número de partes que participan en ella, de la remodelación de las actividades del gobierno, de una mayor conciencia sobre la necesidad de cooperación entre los actores y del desarrollo de nuevos enfoques e instrumentos. Ningún actor por sí solo tiene el conocimiento y la información para solventar problemas complejos, tampoco tiene la perspectiva para utilizar eficientemente los instrumentos necesarios ni el potencial de acción para dominar de manera unilateral (Kooiman, 2004). Es decir, los gobiernos locales deben reforzar la idea de que ellos son los que deben generar los medios para establecer las interacciones sociopolíticas de interdependencia y cooperación entre actores. 
La planeación estratégica y sectorial de las ciudades, y la gestión de redes de actores (con la implicación de la sociedad civil organizada) se han convertido en elementos clave para generar dinámicas que promuevan el involucramiento y la interacción entre actores que fomentan la GP. Los estudios de Pascual i Esteve (2007) dicen que los planes estratégicos suelen crear asociaciones o fundaciones que ayudan a su elaboración y coordinación entre actores, lo que permite desarrollar procesos que conllevan a un mejor entendimiento de las necesidades y a una mayor articulación entre las políticas urbanas. Sin embargo, estos procesos requieren de liderazgos y representatividades muy claras, de la definición de las atribuciones y competencias de los diferentes actores, y del establecimiento de las formas y normas de organización y operación, así como de metodologías para la participación.

Los planes estratégicos promueven el desarrollo humano, integrando, además de los objetivos económicos, sociales, ambientales y territoriales, valores y nuevas formas de gobernar y gestionar las relaciones sociales en el territorio. Por su parte, las redes de actores buscan ser inclusivas, transparentes y participativas, pero no debemos omitir que, a veces, pueden ser riesgosas y servir a los grupos más poderosos. En este último sentido, algunas redes de gobernanza son muy restrictivas en su acceso, y muy elitistas, opacas en su funcionamiento y poco responsables hacia el público en general (Blanco, 2009). Por lo anterior, el impulso a la madurez social participativa podría frenar los procesos discrecionales, con frecuencia comunes al interior de las administraciones públicas, que suelen temer la pérdida de control del rumbo de la sociedad, aunque, como lo señala Mayntz (2001), la gobernanza basada en la negociación entre los actores políticos y sociales no es una pérdida de control del Estado, sino un cambio de forma del gobierno, donde se delega la decisión a instituciones de autorregulación social y el Estado conserva el derecho de la ratificación legal y de la intervención mediante acciones legislativas o ejecutivas, en caso de que la autorregulación demuestre ser ineficaz. Ambos, planes y redes, sirven como instrumentos de cambio.

Estas condiciones abren la posibilidad de generar nuevas lógicas de transversalidad interna en la administración local y nuevos esquemas de cooperación multinivel entre las distintas escalas de gobierno. En este último aspecto, la descentralización de la administración pública es de gran ayuda, ya que los gobiernos locales son el centro de la actividad de la GP al constituirse como espacios donde se facilita la participación y desde donde se pueden generar impactos cercanos a los habitantes (Martínez, 2014). Es decir, la GP significa un tipo de reforma que intenta hacer más eficiente y democrático el Estado, donde, de acuerdo con Aguilar (2006), hay una estructura institucional y técnica enfocada en el desarrollo de actividades (normas, políticas, presupuestos, servicios, etc.) para construir socialmente, en interdependencia, asociación y corresponsabilidad con el Gobierno, la dirección de la sociedad.

\section{Gobierno abierto: transparentando procesos}

Ante la fuerte desconfianza de los ciudadanos sobre la falta de transparencia de la gestión pública, ocasionada por los múltiples casos de discrecionalidad en el uso de los recursos públicos, la nueva gobernanza exige procesos transparentes donde los ciudadanos puedan interactuar con los gobiernos. En consecuencia, se ha tratado de fortalecer la gestión pública a través de la creación de mecanismos y de instituciones para dar transparencia y mejorar los procesos decisionales. Una de las acciones más notables ha sido el fomento al llamado gobierno abierto (GA). Su referente actual más importante es la iniciativa internacional multilateral, surgida en 2011, Open Gouvernement Partnership (OGP), cuyo objetivo es asegurar compromisos concretos de los gobiernos 
participantes — 70 en total, entre ellos España—, para promover la transparencia, empoderar a los ciudadanos, luchar contra la corrupción y aprovechar las nuevas tecnologías para fortalecer la gobernabilidad. Además, el OGP es supervisado por un comité directivo que incluye representantes de gobiernos y organizaciones de la sociedad civil (OGP, 2018).

El GA es un constructo que se encuentra en su fase emergente y en el cual los estudios de Criado y Ruvalcava (2016) demuestran que en Espańa hay un fuerte enfoque tecnológico y social, y que, en términos de participación ciudadana, las plataformas digitales — como páginas web y redes sociales - son las herramientas más utilizadas para la participación.

El GA también es una filosofía político-administrativa, un nuevo paradigma basado en una interacción sociopolítica con valores y principios de transparencia, democracia participativa, empoderamiento, rendición de cuentas, open data y el uso de tecnologías. A partir de ello, se construyen estrategias para el diseño, la implementación, el control y la evaluación de las políticas públicas y para la modernización administrativa, ubicando al ciudadano en el centro de la atención y como una prioridad (Cruz-Rubio, 2015). Es decir, el GA se convierte en una herramienta institucional significativa para el desarrollo de la nueva gobernanza.

Aunque el GA es producto de un debate histórico por el derecho de los ciudadanos a tener acceso a la información producida por el Gobierno y busca, a través del acceso libre a la información, promover procesos más democráticos y transparentes que permitan una relación de confianza entre el Gobierno y los ciudadanos (Sandoval-Almazán, 2015), y, así, ser una herramienta fundamental para los procesos participativos mediante la promoción de alianzas estratégicas (Acosta y
Plata, 2016), todavía es percibido por los funcionarios públicos, en muchos casos, como un ejercicio de transparencia, más que de colaboración y coproducción.

Estas características y condicionantes necesarios para transitar hacia una nueva gobernanza suelen desarrollarse en función de la experiencia, responsabilidad y madurez político-social en materia participativa, como lo muestra el caso de Barcelona, que a continuación se presenta.

\section{Barcelona y su experiencia participativa}

A pesar de la dificultad que representa superar la tradición vertical y centralista decisional, Barcelona es una de las ciudades vanguardistas en impulsar el cambio de paradigma para transitar hacia una nueva gobernanza con modelos decisionales más participativos e inclusivos. Borja (2013) afirma que "en Barcelona existe una sociedad civil implicada en el urbanismo y en la calidad de la ciudad" (p. 179) desde los años 70. Las primeras propuestas de participación ciudadana en el ámbito urbano se desarrollaron con la elaboración de los planes urbanísticos populares (el plan popular de Nou Barris, el plan popular del Casc Antic, etc.), sentando las bases para la negociación en materia urbana, pero sin contar con canales institucionales, principalmente para los Planes Especiales de Reforma Interior (PERI) de mediados de los años ochenta. Este incipiente urbanismo participativo entró en crisis con la aparición de los primeros ayuntamientos democráticos y con el consecuente proceso de cooptación de dirigentes vecinales y del conocimiento experto por parte de los partidos políticos que representaban las nuevas instituciones. En esta ciudad, se impuso la democracia representativa, en la que la participación se hacía por invitación y la administración pública acordaba previamente los contenidos, formatos y actores. 
En 1986, se realizaron las primeras normas de participación ciudadana, en las que se institucionaliza el sistema de consejos consultivos sectoriales, pero predominaron los criterios de racionalidad técnica en el urbanismo y, en ocasiones, estos daban lugar a prácticas de clientelismo asociativo (Bonet, 2014). No obstante, estas normas permitieron impulsar la participación social al descentralizar la administración pública a través de los distritos y sus barrios; este hecho abrió el camino para integrar actividades en los barrios a través del acercamiento entre la administración y la generación de políticas que respondían más a las necesidades de la clase trabajadora. De esta manera, se promovió la participación ciudadana, facilitando las relaciones con la comunidad e incrementando las oportunidades para promover el contacto con los habitantes (Borja, 1996).

Podemos decir que desde hace dos décadas se fortaleció en Barcelona la institucionalización de la participación a través del desarrollo y enriquecimiento de su marco jurídico a nivel local y autonómico.

\section{La institucionalización de la participación}

La legislación española en materia de participación ciudadana ha adquirido diversas modalidades y formas de aplicación en las diversas comunidades autónomas. La crisis económica, social y política española del 2008 impactó a las políticas de participación ciudadana, de manera que se adecuaron los instrumentos participativos a la nueva realidad, simplificando y haciendo más operativas las estructuras formales para impulsar procesos participativos (Diputació de Barcelona, 2017b) ${ }^{1}$.

El caso catalán se ha caractetizado, de acuerdo con Castel Gayán (2011), por fomentar una polítca artesanal o experimental, de naturaleza político-estratégica, que no busca tanto la aprobación de una norma jurídica, sino la experimentación con procesos y ámbitos que generen una nueva cultura política de participación de carácter deliberativo. Hay que recordar que el carácter deliberativo de la participación radica en el fomento de una política democrática basada en la deliberación pública sobre el bien común, la cual requiere de alguna forma de igualdad manifiesta entre los ciudadanos y conforma su identidad y sus intereses, contribuyendo, así, a la concepción pública del bien común. Para lograr dicha igualdad manifiesta, el desarrollo de instituciones y normativas para la toma de decisiones colectivas juega un rol primordial y deberá garantizar que la deliberación sea libre, razonada, con igualdad de estatus de los participantes en cada etapa del proceso deliberativo y con la posibilidad de alcanzar un consenso (Cohen, 1989).

Particularmente en Barcelona, las leyes e instituciones que impulsan la creación de condiciones para la participación ciudadana y que promueven instrumentos y acciones conducentes para la práctica de los principios de la nueva gobernanza han evolucionado de manera significativa, principalmente en las últimas administraciones. Con la Ley 2/2002 de urbanismo, se facultó, sin carácter de obligatoriedad, a los ayuntamientos para crear consejos asesores urbanisticos, y con la Ley de Urbanismo 305/2006 se regula el derecho a la información y a la participación ciudadana en la actividad urbanística (Diputació de Barcelona, 2011). Ambas leyes sirvieron como base para incentivar la participación social y alentaron la creación de diferentes herramientas (manuales, metodologías, guías operativas, etc.) capaces de conducir la participación ciudadana. Sin embargo, con la aprobación y puesta en marcha del Reglamento de participación ciudadana (2017), se depuraron los procesos y se amplió la visión de la participación bajo los principios de la nueva gobernanza. Este reglamento tiene como objetivo “desarrollar las previsiones de la Carta Municipal 
de Barcelona $^{2}$ con respecto a la participación ciudadana y regular los canales de relaciones entre la ciudadanía y el Ayuntamiento para facilitar y promover esta participación en los procesos de toma de decisiones políticas y en la gestión de los servicios y los asuntos de interés municipal” (Diputació de Barcelona, 2017b, p. 14). Además, este reglamento promueve la creación de las condiciones que incentivan el ejercicio del derecho a la participación de manera inclusiva y deliberativa, así como la creación de los medios, canales e instrumentos que facilitan el acceso a la información y comunicación entre los diferentes actores involucrados. No obstante, "no permite una vinculación directa sobre los poderes públicos, ya que sus resultados, por imperativo legal, siempre tienen carácter consultivo" (Diputació de Barcelona, 2017b, p. 8), es decir, la participación aún es de carácter consultivo y puede influir la decisión, pero no es determinante.

La tradición participativa de Barcelona, reforzada por el marco legal, llevó a sus instituciones a crear los órganos de participación y espacios participativos para debatir y recoger las opiniones y propuestas de los barceloneses, así como para elaborar informes o dictámenes sobre proyectos de actuación o sobre actuaciones ya desarrolladas y colaborar con el desarrollo de alguna actuación (coproducción). Los órganos de participación, según el Reglamento de participación (2017), pueden ser de carácter territorial y sectorial. Estos se caracterizan por ser "los medios de encuentro regular entre la ciudadanía y Ayuntamiento para debatir y recoger, de manera continuada, sus opiniones y propuestas sobre las actuaciones municipales" (Diputació de Barcelona, 2017b, p. 35). Cuando estos órganos son de carácter territorial, sirven para la discusión de las políticas públicas que motivan el desarrollo territorial en sus difererntes escalas, y son sectoriales cuando sus funciones se relacionan con una determinada esfera funcional de la actuación municipal o con algún equipamiento o servicio público municipal.

Los órganos de participación territorial son el Consejo de ciudad, los consejos ciudadanos de distrito, los consejos de barrio y las audicencias públicas de distrito (tabla 1). Estos órganos están pensados para las diferentes escalas territoriales y tratan de llegar hasta la más cercana al habitante: el barrio; sin embargo, existen más de 500 órganos de participación en Barcelona, cuya actividad es estrictamente formal, ya que se reúnen solamente cuando el ayuntamiento los convoca, derivando en la pérdida de contenido y efectividad, debilitándolos hasta, algunas veces, inactivarlos (Pindado, comunicación personal, 2 de octubre, 2016). Un ejemplo de ello es la Comisión de seguimiento del Plan de Barrios en la Barceloneta, que ahora se encuentra inactiva. Esta comisión se creó "debido al alto grado de formalidad del Comité de evaluación de seguimiento y al alto número de personas de otras administraciones que lo integran. [Ante la multiplicidad de personas] algunas entidades y asociaciones del barrio muy a menudo sienten que no es un lugar cómodo para expresarse y les resulta difícil manifestar sus inquietudes y opiniones en este órgano" (Pindado, comunicación personal, 2 de octubre, 2016). Finalmente, la comisión fue sustituida por la Comisión de seguimiento del Consejo de barrio para no duplicar estructuras.

Asimismo, se debe considerar que el presupuesto promedio anual para la participación social es de 1 millón de euros, destinados a mantener a los consejos de participación y apoyar a los distritos en sus procesos participativos, es decir, se mantienen estructuras y procesos, de manera que su rentabilidad social puede ser muy discutible. 
Tabla 1. Órganos de participación territorial en Barcelona

\begin{tabular}{|c|c|c|}
\hline Órgano de participación & Objetivo & Sectores participantes \\
\hline $\begin{array}{l}\text { Consejo de ciudad } \\
\text { Máximo órgano consultivo y de participación }\end{array}$ & $\begin{array}{l}\text { Asesorar al ayuntamiento en la definición } \\
\text { de las grandes líneas de la política y } \\
\text { gestión municipal (planeación, reglamentos, } \\
\text { ordenanzas, presupuestos y resultados de } \\
\text { indicadores de la gestión municipal) }\end{array}$ & $\begin{array}{l}\text { Representantes del ayuntamiento: } \\
\text { Alcalde, un concejal de cada uno de los } \\
\text { grupos municipales, representantes de cada } \\
\text { uno de los consejos ciudadanos de distrito, } \\
\text { representantes de cada uno de los órganos } \\
\text { de participación de ámbito de ciudad, } \\
\text { representantes de las instituciones más } \\
\text { significativas de la ciudad, representantes } \\
\text { de las asociaciones registradas en el fichero } \\
\text { de entidades ciudadanas, un máximo de } 15 \\
\text { personas de renombre ciudadano, un máximo } \\
\text { de } 25 \text { ciudadanos y ciudadanas, así como } \\
\text { el comisionado en materia de participación } \\
\text { ciudadana. }\end{array}$ \\
\hline $\begin{array}{l}\text { Consejo ciudadano de Distrito } \\
\text { Máximo órgano consultivo y de participación } \\
\text { de distrito }\end{array}$ & $\begin{array}{l}\text { Asesorar al consejo de distrito. Conocer y } \\
\text { debatir el programa de actuación municipal, } \\
\text { ordenanzas y reglamentos generales de } \\
\text { distrito, conocer y debatir presupuestos } \\
\text { municipales y los grandes proyectos del } \\
\text { territorio, y apoyar a los consejos municipales } \\
\text { sectoriales de distrito }\end{array}$ & $\begin{array}{l}\text { Presidente del consejo de distrito } \\
\text { Concejal de distrito } \\
\text { Concejales adscritos al distrito } \\
\text { Consejeros de distrito } \\
\text { Representantes de asociaciones y entidades } \\
\text { de distrito } \\
\text { Ciudadanos escogidos aleatoriamente } \\
\text { Un representante de cada uno de los consejos } \\
\text { sectoriales de distrito, jefe de la secretaría } \\
\text { técnica. }\end{array}$ \\
\hline Consejo de Barrio & $\begin{array}{l}\text { Ser un canal de participación ciudadana en el } \\
\text { desarrollo de políticas públicas de proximidad } \\
\text { y favorecer la cohesión social }\end{array}$ & $\begin{array}{l}\text { Concejal (a) de distrito } \\
\text { Dos vicepresidencias una representada por } \\
\text { un ciudadano(a) y otra por el concejal(a) de } \\
\text { la lista más votada del barrio, un concejal(a) } \\
\text { de distrito de cada grupo municipal y las } \\
\text { entidades, asociaciones, grupos, etc. del } \\
\text { barrio y vecinos en general. }\end{array}$ \\
\hline
\end{tabular}

Queremos destacar al Consejo de barrio, pues este juega un papel primordial en la materialización de las acciones públicas a pequeña escala, ya que tiene como objetivo "ser un canal de participación ciudadana en el desarrollo de políticas públicas de proximidad y conviviencia y favorecer la cohesión social y la mejora de la calidad de vida" (Ajuntament de Barcelona, 2017b). Sus actividades se desarrollan en diferentes equipamientos municipales ubicados en cada uno de los distritos de Barcelona (figura 1).

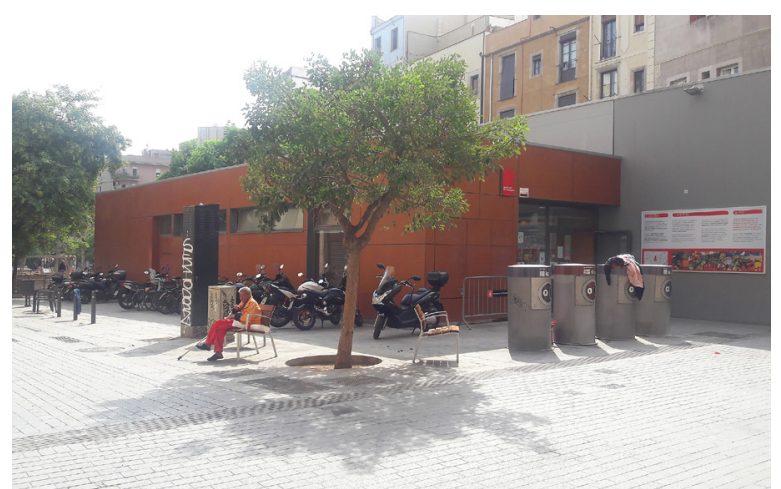

Figura 1. Equipamiento del Ayuntamiento de Barcelona ubicado en el distrito de Ciutat Vella. En él, se realizan diversas actividades para los habitantes del distrito

Fuente: elaboración propia 
Para orientar las acciones en los barrios en materia de educación, derechos sociales, actividad económica y ecología urbana, Barcelona ha desarrollado el Plan de barrios, en el que participan las asociaciones vecinales de cada barrio. Su presupuesto proviene en un 100\% del ayuntamiento, hecho que les da autonomía, y es de 150 millones de euros, los cuales deben ser distribuídos durante un periodo de 4 ańos (Ajuntament de Barcelona, 2017c). La vocación es transversal (se reúnen de manera periódica con los distritos) y busca la implicación ciudadana en la definición, en el seguimiento de los proyectos y en la voluntad e innovación social (se considera que es necesario innovar para desarrollar políticas que disminuyan las desigualdades). Actualmente, existen 10 planes de barrio que afectan a 16 barrios, 6 distritos y 226.691 personas. Los barrios escogidos se caracterizan por tener malas condiciones de salud, estudios, ocupación, vivienda, renta y vulnerabilidad social (Universidad de Barcelona, 2017).

Aunado a ello, existen iniciativas institucionales de trabajo transversal. Así, por ejemplo, con la creación de la Oficina de los derechos de ciudadanía, cultura, participación y transparencia se crean espacios de comunicación y enlace para desarrollar proyectos de acción comunitaria y participación colaborativa que permiten atender problemas comunes y vigilar las acciones gubernamentales para fomentar la transparencia, rendición de cuentas y reforzar la confianza (Diputació de Barcelona, 2017a). Sin embargo, existen dificultades para la coordinación interdepartamental, por lo cual, el actual ayuntamiento firmó el decreto Democracia Activa, a través del cual se creó el Programa de Coordinación Interdepartamental, cuya tarea consiste en servir de enlace entre los departamentos del ayuntamiento que desarrollen trabajos de participación para coordinarlos y ponerse de acuerdo sobre los conceptos, métodos, recursos, sistemas, etc. No obstante, ni las propuestas para mejorar los procesos participativos ni las propuestas ciudadanas pueden garantizar su seguimiento entre una administración y otra.

Por otro lado, los principios del gobierno abierto se tratan de poner en práctica mediante los medios digitales institucionales como el Decidim Barcelona y Govern Obert, a través de los cuales se difunde la información para dar transparencia a los procesos y promover la participación en las redes sociales. En Decidim Barcelona, se pueden identificar procesos de comunicación, diagnóstico, aportaciones, decisiones y resultados, y evaluación de las propuestas (Ajuntament de Barcelona, 2017d). Esta plataforma es uno de los avances más significativos que reconoce el actual ayuntamiento de Barcelona en materia de participación social para llegar a un mayor número de ciudadanos interesados en la participación.

\section{La participación ciudadana desde el barrio}

La escala más aprehensible para desarrollar las prácticas participativas es el barrio y, por esta razón, se mostrarán algunos ejemplos de acciones participativas barriales que ilustran el fomento y las dificultades que surgieron al momento de impulsar la gobernanza participativa en Barcelona.

Para empezar, en la década del 2000 los estudios de Blanco (2009) detectaron una desigual intensidad de la innovación política local en función de los territorios y de las diferentes formas de articular la gobernanza urbana, desde los modelos más bottom-u $p^{3}$, hasta los modelos más top-down. Por ejemplo, en las políticas de regeneración urbana de áreas desfavorecidas de los barrios del Raval (centro histórico) y Trinitat Nova (periferia), los estudios de Blanco (2009) demuestran que en el Raval predominaron los actores institucionales y privados empresariales, mientras que en Trinitat Nova se destacó la participación vecinal y la coordinación horizontal entre los equipamientos y los servicios de proximidad. Estos casos sugieren que distintos modelos de gobernanza pueden asociarse a diversas opciones de política pública. 
En un marco de gobernanza participativa, el escenario ideal es como el de Trinitat Nova, donde se ha ido formando una cultura de la participación que los propios vecinos reconocen, tal como se puede identificar en las expresiones de algunos de ellos. Por ejemplo, cuando la presidenta del barrio dice que "se empieza por la ilusión en querer hacer cosas, en creer que podemos cambiar la realidad y en la confianza en que, aunque está difícil, vale la pena intentarlo" (Pauné, 2015), y cuando el representante de la asociación de vecinos comenta que "aquí la gente es muy participativa y solidaria, organizamos muchas actividades, los vecinos se relacionan a diario y se ayudan" (Pauné, 2015). Sin embargo, a veces se sienten olvidados por el Gobierno local. Otro ejemplo de este tipo es el Barrio del Bon Pastor, donde la junta de vecinos del barrio, junto con el ayuntamiento de Barcelona y la Universidad de Barcelona, están desarrollando un proyecto para construir viviendas baratas y crear un espacio museístico en el Barrio. Desde el 2016, se iniciaron las reuniones para definir cómo será el proyecto y darle un valor histórico a este barrio de tradición industrial. Se realizan actividades participativas con un grupo impulsor que se encarga de procurar coproducir las actuaciones del Plan de Barrio y de definir los grupos de trabajo y los procesos de participación. Este barrio cuenta con un fuerte sentido de participación y sus habitantes se encuentran organizados desde los años setenta. Su compromiso participativo se hace evidente en la expresión de uno de los vecinos de mayor edad, cuando dice que "nosotros entendemos la participación desde la perspectiva de proponer, informar, debatir y sacar conclusiones colectivas de los procesos" (Universidad de Barcelona, 2017). Lo anterior se desarrolla en un marco de cooperación con los ayuntamientos, las universidades o con cualquiera que esté interesado, hecho que facilita la fluidez de los trabajos.

En contraparte, existen lugares donde es difícil la negociación, como en la Barceloneta (del distrito de Ciutat Vella), donde, en 2016, el ayuntamiento de Barcelona propuso, en el marco de las acciones para la recuperación de los espacios públicos, recuperar un baldío (que estaba con acceso cerrado) ubicado en el barrio de la Barceloneta. Para ello, se realizaron reuniones con los vecinos afectados con el fin de hacer el diagnóstico y plantearles la propuesta, la cual sería ejecutada por un grupo de jóvenes arquitectos. Aunque la propuesta en términos de diseño no tuvo grandes inconvenientes, algunos vecinos manifestaron su desacuerdo en recuperar el espacio, pues decían que abrirlo atraería a grupos de jóvenes a divertirse y estos suelen ser ruidosos y, a veces, se emborrachan. Debido a que este hecho perturbaría la tranquilidad de los vecinos, algunos de ellos preferían que el baldío se quedara sin intervenir. Esta situación creó ciertas tensiones entre los participantes (figura 2). Aunque las autoridades tomaron nota de las observaciones de los participantes, comentaron que el trabajo se iniciaría a principios del 2017 , debido a la temporalidad de sus procesos administrativos y del riesgo de perder el presupuesto. Esto pone en evidencia la dificultad para coordinar las lógicas administrativas con las participativas, así como la imperante decisión política ante los largos procesos de negociación a los que puede llevar la participación social.

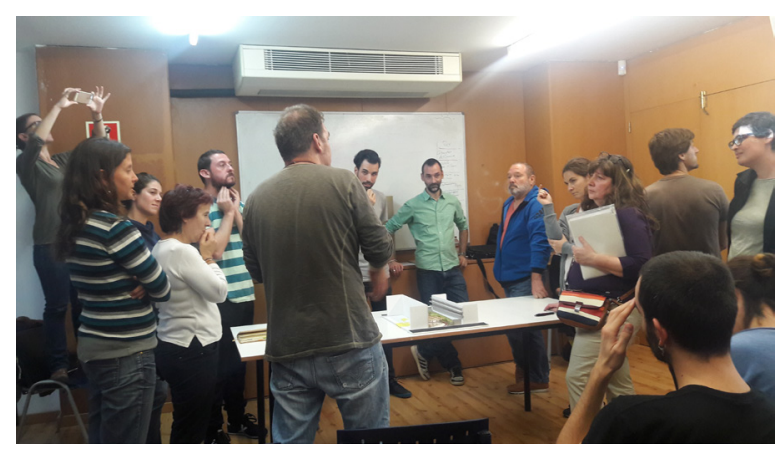

Figura 2. Reunión de funcionarios con los vecinos de la Barceloneta para la presentación de la propuesta del jardín con el objetivo de recuperar un espacio público abandonado

Fuente: elaboración propia

Finalmente, el proyecto se ejecutó en el 2018 y fue bien aceptado, ya que se pusieron cámaras de 
vigilancia y un horario de apertura y cierre del parque para garantizar la seguridad y la tranquilidad de los habitantes. Aunque el diseño fue cuestionado por algunos vecinos, resultó ser un éxito para los niños (figura 3). El comentario de una vecina lo deja en claro, cuando dide que "cuando lo vi [el jardín] dije ¡Uy! Qué cosa más fea nos han puesto, jtodo lleno de cemento! Aunque no es cemento, es un material blando, y mira, mi hijo se la pasa muy bien. Me gusta que lo hayan recuperado" (S. García, comunicación personal, 30 de abril de 2019). Esta situación constata el efecto positivo de los procesos participativos, ya que las tensiones se tradujeron en propuestas que, al ser tomadas en cuenta por parte de los funcionarios públicos, coadyuvan a la aceptación del proyecto, sin llegar a decisiones radicales y evitando que este espacio permaneciera cerrado y sin intervención, es decir, fue un ganar-ganar.

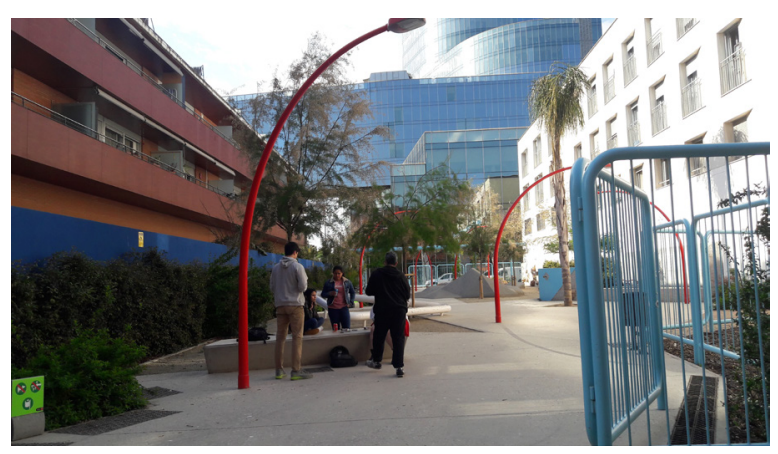

Figura 3. Espacio público intervenido, Ilamado Jardín D’ Antònia Vilàs I Ferràndiz

Fuente: elaboración propia

\section{La participación ciudadana desde las plataformas digitales}

Uno de los medios para la participación ciudadana que puede tener mayor cobertura es el uso de las plataformas digitales. En esta materia, una de las experiencias recientes (inscrita en los principios del gobierno abierto), que permitió enriquecer de alguna manera la decisión política, fue la elaboración del Plan de Actuación Municipal
$(\mathrm{PAM})^{4}$ en el 2016. El proceso participativo, con una duración de dos meses, fue de carácter híbrido, ya que se establecieron medios presenciales y digitales (plataforma oficial y redes sociales) para su desarrollo. La participación, calificada en general como "positiva" por los funcionarios, fue de más de 42.000 personas, el $43 \%$ por medios presenciales y el $57 \%$ por medios digitales ${ }^{5}$ (Ajuntament de Barcelona, 2017d). Además, se contrataron empresas para que contactaran a los principales grupos sociales, de acuerdo con las temáticas de los debates. La plataforma digital permitió recibir las propuestas por temática, contabilizarlas, someterlas a votación virtual, y conocer y contabilizar los comentarios. Sin embargo, es necesario hacer estudios para revisar sus metodologías y evaluar los márgenes de error, así como para nalizar la eficacia del proceso y su influencia en la decisión gubernamental. Aunque este tipo de herramients intentan llegar al mayor número de habitantes posible, todavía es difícil acceder a los grupos más vulnerables.

Estos breves ejemplos nos muestran el interés público y ciudadano que existe para avanzar en la construcción de una nueva gobernanza donde se intercalen modelos de gobernanza mixtos experimentales y donde principios como la inclusión y la deliberación se han ido desarrollando desde su marco normativo y la praxis. Estos ejemplos también revelan la importancia del desarrollo de espacios físicos y virtuales que procuren la interacción entre los actores y que influyan en el fomento de una cultura participativa. No obstante, se observa que uno de sus mayores retos es el de llegar al mayor número de habitantes en los procesos participativos. En este sentido, las plataformas digitales representan una herramienta útil para difundir la información y fomentar la inclusión en los procesos participativos. Sin embargo, estas se encuentran en una etapa emergente y deberán ser sometidas a revisiones profundas. 


\section{Conclusión}

El mandato internacional para impulsar la participación ciudadana en las nuevas formas de gobernanza desde los gobiernos locales ha sido acogido por muchas ciudades desde sus políticas públicas y marcos jurídicos. Barcelona es un claro ejemplo de los esfuerzos que se han llevado a cabo para construir una gobernanza participativa (GP) basada en la experimentación y retroalimentación de sus procesos, demostrando que la escala barrial es la base para fomentar acciones participativas y que su desarrollo debe contar con instituciones y normativas que permitan crear las condiciones técnico-administrativas, presupuestales, organizacionales y comunicacionales (entre las más destacadas) que faciliten las interacciones en condiciones de igualdad entre los actores involucrados. Esto le da un impulso a la coordinación y a los acuerdos sobre aspectos conceptuales, metodológicos y procedimentales, entre otros, que son útiles para la búsqueda de objetivos comunes que permitan construir y reconstruir la ciudad a través del mejoramiento de las políticas públicas.

Avanzar hacia una GP ha significado para los barceloneses enfrentar dificultades y ganar experiencia en los procesos, reconociendo que en un ámbito de transición, donde se incentiva el enriquecimiento decisional a través del debate colectivo, es común que las contradicciones desaten conflictos, que pueden ser aprovechados para repensar y alimentar las decisiones. El caso barcelonés nos muestra que la participación es un trabajo constante que permite construir estructuras duraderas desde las instituciones en colaboración con los habitantes, para avanzar en la sustitución de los sistemas decisionales jerárquicos hacia los de gobernanza participativa. Para ello, es necesario que los instrumentos normativos evolucionen para que puedan dar mayores garantías que aseguren la continuidad de los procesos entre una administración y otra, así como otras que permitan buscar mejores estrategias que ayuden al Gobierno en el desarrollo de acciones facilitadoras para incentivar la participación de los grupos más vulnerables y para garantizar la mayor influencia en las decisiones políticas a partir del diálogo y la negociación entre los actores. Por otro lado, la incipiente y útil práctica de los principios técnicos del gobierno abierto ayudan a incentivar la participación y a vigilar los procesos para que no se privilegien los intereses individuales sobre los colectivos. Al mismo tiempo, uno de los grandes desafíos para el gobierno abierto es asegurar la inclusividad y la equidad entre los actores y garantizar la transparencia y la veracidad durante los procesos y la rendición de cuentas.

Podemos decir que la construcción de una nueva gobernanza en Barcelona está en un momento de búsqueda de innovación de sus procesos participativos para hacerlos más deliberativos y ayudarlos a mejorar en la construcción de su cultura participativa.

La experiencia de Barcelona es una evidencia de que la gobernanza participativa representa un cambio de paradigma hacia nuevas formas de pensar y repensar las ciudades, el cual es un gran reto conceptual, tecnológico, instrumental y operacional que permite avanzar hacia una nueva forma de pensamiento colectivo capaz de transitar de una nueva gobernanza a una nueva forma de vida en sociedad. Esto es, sin duda, un reto a largo plazo.

\section{Agradecimientos}

A la Vicerrectoría de Investigación y Estudios de Posgrado de la Benemérita Universidad Autónoma de Puebla, México. A los profesores Josep Roca Cladera y Blanca Arellano Ramos, del Centro de Política de Suelo y Valoraciones (CPSV) de la UPC. A Fernando Pindado Sánchez y Belén García Quiñones, funcionarios del ayuntamiento de Barcelona. 


\section{Referencias}

Acosta, S. I., y Plata O. J. (2016). Participación, colaboración, transparencia y datos abiertos. Tras la definición de Gobierno abierto. Teuken Bikiday, 7(9), 209-222. Recuperado de http:// revistas.elpoli.edu.co/index.php/teu/article/ view/1016/840

Aguilar, L. (2006). Gobernanza y gestión pública. México: Fondo de Cultura Económica.

Ajuntament de Barcelona. (2017a). Reglamento de participación ciudadana. Recuperado de https:// ajuntament.barcelona.cat/participaciociutadana/ sites/default/files/documents/reglamento_participacion_cast.pdf

Ajuntament de Barcelona. (2017b). Derechos de ciudadania, cultura, participación y transparencia. Recuperado de http:// governobert.bcn.cat/es/participacion/ espacios-estables-de-participaci\%C3\%B3

Ajuntament de Barcelona. (2017c). El pla dels barris de Barcelona. Recuperado de http://pladebarris.barcelona/

Ajuntament de Barcelona. (2017d). Programa de Actuación Municipal. Recuperado de https:// www.decidim.barcelona/processes/pam?locale=es

Blanco, I. (2009). Gobernanza urbana y políticas de regeneración: el caso de Barcelona. Revista Española de Ciencia Politica, (20), 125-146. Recuperado de https://recyt.fecyt.es/index.php/ recp/article/view/37478/20996

Bonet, J. (2014). La participació ciutadana en l'urbanisme: potencials i limits. Papers. Regió $\mathrm{Me}$ tropolitana de Barcelona, (57), 63-70. Recuperado de https://ddd.uab.cat/record/117944
Borja, J. (1996). The city, democracy and governability: the case of Barcelona. En UNESCO, International Social Science Journal. Democracy and Governance of the City. (pp. 8593). UK: Blackwell Publishers. https://doi. org/10.1111/j.1468-2451.1996.tb00059.x

Borja, J. (2013). Revolución urbana y derechos ciudadanos. Madrid, España: Alianza Editorial.

Callon, M. (1997). Concevoir: Modèle hiérarchique et modèle négocié. En Lélaboration des projets architecturaux et urbains en Europe. Vol I. Les acteurs du projet architectural et urbain. Plan construction et architecture. (pp. 169-174). París : Francia.

Castel Gayán, S. (2011). Descentralización política, participación ciudadana y renovación jurídica ¿hacia una democracia participativa? Revista catalana de dret públic. (43), 279-316. Recuperado de http://revistes.eapc.gencat.cat/index.php/ rcdp/article/view/2233

Cohen, J. (1989). Deliberación y legitimidad democrática. Cuaderno Gris. Época III, 9, $127-$ 145. Recuperado de https://repositorio.uam.es/ bitstream/handle/10486/361/21813_Deliberaci\%F3n\%20y\%20legitimidad\%20democratica. pdf?sequence $=1$

Comunidad Autónoma de Cataluña. (2014). Ley 22/1998, de 30 de diciembre, de la Carta Municipal de Barcelona. Texto Consolidado. Boletín Oficial del Estado. Legislación Consolidada.

Criado, I., y Ruvalcava, E. (2016). ¿Qué es y qué se entiende por gobierno abierto? Análisis de la percepción en implementación del Gobierno Abierto en el ámbito local español. Madrid: NovaGob y Universidad Autónoma de Madrid. 
Cruz-Rubio, C. (2015) ¿Qué es (y qué no es) gobierno abierto? Una discusión conceptual. Economía. Revista en Cultura de la Legalidad. (8), 37-53. Recuperado de https://e-revistas.uc $3 \mathrm{~m}$.es/index.php/EUNOM/article/ download/2475/1359

Diputació de Barcelona. (2011). Urbanisme i participació: iniciatives i reptes de futur. Conclusions del grup de treball sobre urbanisme i participació ciutadana en l'àmbit local. Barcelona: Colección, Documentos de Trabajo. Recuperado de http:// www.gea21.com/archivo/pb-urbanisme-i-participacio-iniciatives-i-reptes-de-futur/

Diputació de Barcelona. (2017a). Servei de Convivència, Diversitat i Participació Ciutadana. Recuperado de https://www.diba.cat/web/ acciocomunitariaparticipacio/presentacio.

Diputació de Barcelona. (2017b). Reglament tipus de participación ciutadana. Collecció, Eines. Sèrie Benestar i ciutadania. Recuperado de http:// www1.diba.cat/libreria/pdf/58846.pdf

Kooiman, J. (2004). Gobernar en gobernanza. Revista Instituciones y Desarrollo. (16), 171-194. Recuperado de https://www.researchgate.net/publication/39201587_Gobernar_en_gobernanza

Lefebvre, H. (1975). El derecho a la ciudad (3. ${ }^{\text {a }}$ ed.). Barcelona, España: Península. (Trabajo original publicado en 1967).
Martínez, G. (2014). Gobernanza participativa y reforma del Estado. Recuperado de http://www. academia.edu/6290460/Gobernanza_Participativa_y_Reforma_del_Estado

Mayntz, R. (2001). El Estado y la sociedad civil en la gobernanza moderna. Reforma y Democracia. (21), 1-8.

Open Gouvernement Partnership. (2018). Acerca de OGP. Recuperado de https://www.opengovpartnership.org/about/about-ogp

Pascual i Esteve, J. (2007). La estrategia territorial como inicio de la gobernanza democrática. Los planes estratégicos de segunda generación. Colección, Documentos de Trabajo. Serie, Desarrollo económico. Barcelona, España: Diputació de Barcelona.

Pauné, M. (2015). Vivir en Trinitat Nova, el barrio más pobre de Barcelona. La Vanguardia. Recuperado de http://www.lavanguardia.com/local/ barcelona/20150107/54423032265/trinitat-nova-barrio-mas-pobre-barcelona.html

Sandoval-Almazán, R. (2015). Gobierno abierto y transparencia: construyendo un marco conceptual. Convergencia, 22(68), 203-227. https://doi. org/10.13140/RG.2.1.4760.0802

Universidad de Barcelona. (2017). Gobernanza urbana y participación ciudadana en el curso de UIU Smart Cities. Recuperado de http://www. ub.edu/escult/index.html

Oriol Nello, presidente del consejo asesor del plan de barrios, declaró que entre las pocas cosas buenas de la crisis económica española se encuentra el aumento de iniciativas ciudadanas (Ajuntament de Barcelona, 2017b).

En la Carta Municipal Ley 22/98, de 30 de diciembre (última modificación 30 diciembre 2014), se promueven normas e institutos para facilitar la participación ciudadana que permiten reforzar la práctica democrática y estimular la cooperación entre el ayuntamiento, el movimiento asociativo y los agentes sociales.

Como el caso de Trinitat Nova, que fue inclusivo y plural, o el de El Raval, que fue restrictivo y elitista.

EI PAM es un instrumento municipal que se elabora al inicio de cada mandato y tiene la finalidad de establecer las líneas prioritarias, los objetivos y las actuaciones gubernamentales (Ajuntament de Barcelona, 2017a).

En la plataforma se registraron 197.000 visitas, pero solo se validaban las de los usuarios que comprobaban que eran catalanes (Fernando Pindado, comunicación personal, 2 de octubre, 2016). 